Heijer, C.D.J. den, Bijnen, E.M.E. van, Paget, W.J., Stobberingh, E.E. Fusidic acid resistance in Staphylococcus aureus nasal carriage strains in nine European countries. Future Microbiology: 2014, 9(6), 737-745

\begin{tabular}{|l|l|}
\hline $\begin{array}{l}\text { Postprint } \\
\text { Version }\end{array}$ & 1.0 \\
\hline Journal website & http://www.futuremedicine.com/doi/abs/10.2217/fmb.14.36? \\
\hline Pubmed link & $\underline{\text { http://www.ncbi.nlm.nih.gov/pubmed/25046521 }}$ \\
\hline DOI & $10.2217 / \mathrm{fmb} .14 .36$ \\
\hline
\end{tabular}

This is a NIVEL certified Post Print, more info at http://www.nivel.eu

\title{
Fusidic acid resistance in Staphylococcus aureus nasal carriage strains in nine European countries
}

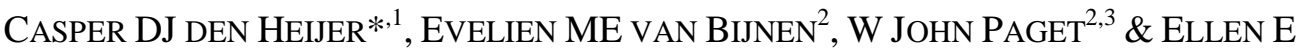 \\ STOBBERINGH ${ }^{1}$ \\ ${ }^{1}$ Department of Medical Microbiology, Maastricht University Medical Centre/CAPHRI, \\ Maastricht, The Netherlands \\ ${ }^{2}$ NIVEL, The Netherlands Institute for Health Services Research, Utrecht, The Netherlands \\ ${ }^{3}$ Department of Primary \& Community Care, Radboud University Medical Centre, Nijmegen, \\ The Netherlands \\ *Author for correspondence: casper.den.heijer@mumc.nl \\ Sections:
}

\begin{abstract}
:
Aim: To evaluate fusidic acid resistance pheno- and geno-typically in nasal carriage Staphylococcus aureus isolated from general practice patients in nine European countries. Materials \& methods: Phenotypic fusidic acid resistance was determined by disc diffusion and MIC values, and genotypically by a PCR detecting fusA-E genes. The main analysis was performed on methicillinsusceptible $S$. aureus strains, because methicillin-resistant $S$. aureus isolates were scarce. Results: Overall S. aureus fusidic acid resistance was low $(<10 \%)$. fus $C$ was the predominant mechanism of resistance and associations were found between resistance genes and specific spa types. Conclusion: S. aureus strains showed low resistance to fusidic acid, making this antibiotic an appropriate choice in the treatment of methicillin-susceptible $S$. aureus skin infections in general practice patients in the nine participating European countries.
\end{abstract}

Staphylococcus aureus is frequently encountered as the causative bacterial pathogen of skin and skin structure infections (SSSIs), such as impetigo. For the local treatment of these infections, fusidic acid is regularly prescribed in Europe [1,2]. In addition, this antibiotic was shown to be as effective as linezolid in treating methicillin-resistant S. aureus (MRSA) SSSIs [3]. However, the effectiveness of this treatment is being hampered by the appearance of resistance to this agent among $S$. aureus strains [2]. Fusidic acid is a bacteriostatic antibiotic by interacting with EF-G, inhibiting its release from the ribosome and thus stopping bacterial protein synthesis [1]. Resistance to this antibiotic can be caused by mutations in fusA (the gene encoding EF-G) or by the plasmid-based fusB, fus $C$ or fus $D$ genes, which encode proteins that prevent the interaction of fusidic acid with EF-G or facilitate the 
Heijer, C.D.J. den, Bijnen, E.M.E. van, Paget, W.J., Stobberingh, E.E. Fusidic acid resistance in Staphylococcus aureus nasal carriage strains in nine European countries. Future Microbiology: 2014, 9(6), 737-745

disconnection between these two compounds [4]. In addition, mutations in $r p l F$, which encodes ribosomal protein L6 (i.e., a ribosomal contact area with EF-G), can result in fusidic acid resistance (fusE) [5].

Until recently, the fusB gene was considered to be mainly responsible for the acquired form of fusidic acid resistance [1]. However, the discovery of the fusC gene in 2007 made it clear that a large proportion of the isolates initially tested negative for the known fusidic acid resistance genes were carrying this newly discovered gene [1]. Recent publications show that most fusidic acid-resistant $\mathrm{S}$. aureus strains have acquired fusidic acid resistance, with dominance of fusB or fusC differing per country [2,6-7]. In younger patients with impetigo, the fusB-positive fusidic acidresistant impetigo clone (epidemic European fusidic acid-resistant impetigo clone; EEFIC) has been shown to be highly prevalent $[6,8]$.

Previous studies on S. aureus fusidic acid resistance have mainly focussed on clinical isolates. However, carriage S. aureus strains could offer important insights, because a person's own microbiota is considered to be the reservoir of resistance and nasal colonizing strains have been shown to often be the source of $\mathrm{S}$. aureus infection $[9,10]$.

Within The APRES study, we collected nasal carriage S. aureus strains in nine European countries via national general practice (GP) networks. In this way, we determined the prevalence of fusidic acid resistance in these countries and investigated the genetic determinants (fusA-E) for this resistance. In addition, we related the genotypic characterization to background information from the patients from whom the isolates originated. Finally, we assessed the clonal relationship between the fusidic acid-resistant $\mathrm{S}$. aureus by means of spa typing, because both fusB and fusC have been linked to specific spa types [6,11]. For all our objectives, we stratified our results by methicillin-susceptible S. aureus (MSSA) and MRSA, because of previously observed differences in fusidic acid resistance genotypes [12].

\section{MATERIALS \& METHODS}

The APRES study design has been described in a previous publication [13]. For this reason, we shall only briefly mention the methods used in this study.

\section{GPs \& patients}

From existing national GP networks in Austria, Belgium, Croatia, France, Hungary, Spain, Sweden, The Netherlands and the UK the participating GPs ( $n=20$ per country was aimed at) were recruited. Each GP was asked to collect nasal swabs from 200 patients, aged $\geq 4$ years (UK: $\geq 18$ years due to ethical committee constraints), who visited his/her practice for a noninfectious condition in the period from November 2010 to August 2011. Patients were excluded when they had been prescribed an antimicrobial agent or had been hospitalized in the previous 3 months. In addition, immunocompromised patients (e.g., diabetes mellitus) and nursing home residents were excluded. With these exclusion criteria, only 'colonized patients' were included, thereby serving as a representative sample of the general population.

Background information of the participants (i.e., age, sex and whether the patient had a medical history of a chronic skin disease) was noted too. 
Heijer, C.D.J. den, Bijnen, E.M.E. van, Paget, W.J., Stobberingh, E.E. Fusidic acid resistance in Staphylococcus aureus nasal carriage strains in nine European countries. Future Microbiology: ) 2014, 9(6), 737-745

\section{Swabs \& S. aureus isolates}

A charcoal swab (Transystem, cod. 114 C, Copan Italia, Italy) was used to swab both anterior nares of the patients. After a swab was collected, it was sent to one (national) microbiological laboratory, where S. aureus was isolated and identified using a standardized protocol [13].

When all swabs were collected and analyzed by the national laboratory, the detected S. aureus strains were frozen (at $-20^{\circ} \mathrm{C}$ ) and shipped batchwise to the microbiological laboratory of Maastricht University Medical Centre (MUMC), The Netherlands, where the antibiotic resistance testing was performed. Apart from fusidic acid, S. aureus resistance was determined for the following antibiotics: azithromycin, ciprofloxacin, clindamycin, daptomycin, erythromycin, gentamicin, linezolid, oxacillin, tetracycline, trimethoprim-sulfamethoxazole and vancomycin [14].

During the study period, a total of 32,203 analyzable swabs were collected, of which 6953 (21.6\%) yielded S. aureus. Of these, 6905 (99.3\%) were available in Maastricht for antibiotic resistance testing [14]. MRSA was confirmed in 91 of these 6905 S. aureus isolates.

\section{Fusidic acid resistance testing}

Initially, disc diffusion zone diameters were determined for all S. aureus strains, according to the European Committee on Antimicrobial Susceptibility Testing (EUCAST) guidelines (10 $\mu$ g fusidic acid discs, Neo Sensitabs, Rosco Diagnostica, Denmark) [15]. Owing to difficulties in interpreting the zone diameters, for which we contacted EUCAST, we decided to confirm our results (classification: susceptible vs resistant) with broth microdilution MIC values. These were assessed for the isolates with zone diameters $\pm 2 \mathrm{~mm}$ from the proposed cutoff value by EUCAST $(<24 \mathrm{~mm}$ is resistant), thus the isolates with zone diameters between 21 and $25 \mathrm{~mm}$ $[6,15]$. For MIC values, we used a cutoff value of $>1 \mathrm{mg} / \mathrm{l}$ to classify the S. aureus strain to be resistant to fusidic acid, which is in accordance with the EUCAST guidelines [15].

In order to evaluate the effect of the specific fusA mutations on the level of resistance, E-tests (BioMérieux, AB Biodisk, Solna, Sweden) were performed on all fusA-positive isolates.

\section{PCR}

All S. aureus classified as fusidic acid resistant, based on the MIC value, were evaluated for the presence of acquired resistance genes (fusB, fusC and/or fusD) in a multiplex PCR approach, as previously described [7]. When strains showed negative results, they were further investigated for chromosomal mutations in fusA and fusE, as described by Castanheira et al. [7]. The obtained nucleotide sequences were aligned using the program ClustalW (Bioinformatics Center, Kyoto, Japan) and subsequently converted into amino acid sequences. With the program Clustal Omega (EMBL-EBI, Hinxton, UK) the amino acid sequences were compared with the wildtype gene retrieved via BLAST [16]. 
Heijer, C.D.J. den, Bijnen, E.M.E. van, Paget, W.J., Stobberingh, E.E. Fusidic acid resistance in Staphylococcus aureus nasal carriage strains in nine European countries. Future Microbiology: 2014, 9(6), 737-745

\section{spa typing}

For feasibility reasons, approximately half of all fusidic acid-resistant strains were randomly selected via the program SPSS version 16.0 (SPSS Inc., IL, USA) for spa typing. In accordance with previous studies, real-time amplification of the spa locus, followed by sequencing, was performed [17]. The algorithm based upon repeat pattern (BURP) with the Ridom StaphType version 1.5 (Ridom GmbH, Münster, Germany) software package [18] was used to cluster the spa types into spa clonal complexes (spa-CCs). We used the recommended settings by the manufacturer.

\section{Statistical analysis}

Statistical analysis was only performed on the MSSA data, because the low numbers of MRSA did not allow for meaningful analysis.

The groups divided by the different fusidic acid resistance genes found were compared with respect to patient characteristics, that is, age (in years), gender (\% women) and a history of a chronic skin disease (yes/no), and bacterial characteristics, that is, disc diffusion zone diameter (in $\mathrm{mm}$ ), proportion multidrug resistant and spa type.

In order for a strain to be classified as multidrug resistant, it should be resistant to three or more antibiotic classes. Penicillin resistance was not included in this classification, because most $\mathrm{S}$. aureus express resistance to this antibiotic.

For the comparison of two groups, $\chi 2$ tests were used for categorical variables; for continuous variables the independent sample t-test or Mann-Whitney U test was performed, dependent on whether parametric assumptions were met. When more than two groups were compared, logistic regression analyses were performed for categorical variables and the analysis of variance (ANOVA) test or Kruskal-Wallis test were used for continuous variables, where appropriate. All statistical analyses were done using SPSS version 20.0 and a p-value of $<0.05$ was considered to be statistically significant.

\section{RESULTS}

\section{Determination of fusidic acid resistance cutoff}

The strains with a zone diameter $\leq 22 \mathrm{~mm}$ all showed a MIC value $>1 \mathrm{mg} / \mathrm{l}$, whereas the isolates with larger zones all showed MIC values below this cutoff value. In this way, we concluded that in our collection the isolates with a zone diameter $<23 \mathrm{~mm}$ should be classified as fusidic acid resistant.

\section{Fusidic acid resistance}

In total, 2.8\% $(\mathrm{n}=194)$ of the MSSA isolates showed resistance to fusidic acid, which ranged from $0.1 \%(n=1)$ in Croatia to $7.8 \%(n=62)$ in the UK (Table 1$)$. In all countries, more than half of the resistant isolates showed acquired fusidic acid resistant genes (fusB, fusC or fusD): range 52.6\% (Belgium) to 100\% (Austria, Hungary). fusC was the most prevalent resistance mechanism $(45 \%$; $n=88)$ and no S. aureus had acquired the fusD gene or showed fusE mutations. Six strains showed 
Heijer, C.D.J. den, Bijnen, E.M.E. van, Paget, W.J., Stobberingh, E.E. Fusidic acid resistance in Staphylococcus aureus nasal carriage strains in nine European countries. Future Microbiology: 2014, 9(6), 737-745

negative results for all resistance genes (four UK, one Belgian and one Dutch isolate).

Nine of the 91 (9.9\%) MRSA isolates were fusidic acid-resistant, of which six originated from France (2 fusA and 4 fusC), and one strain from Austria (fusC), The Netherlands (fusB) and the UK (fusC) each.

\section{fusA mutations \& MIC values}

The mutation most frequently observed among our fusA-positive isolates $(\mathrm{n}=52)$ was the substitution of valine with isoleucine at position 90 (V90I) of the fusA gene ( $n=17$ ) followed by the substitution of leucine by serine at position 461 (L461S, $n=$ 16) (Table 1). These prevalent mutations were accompanied by relatively low MIC values as measured by E-test: V90I, range 2-16 mg/l; L461S, range: 6-24 mg/l. The highest resistance values ( $>256 \mathrm{mg} / \mathrm{l})$ were observed for the following mutations: L461K, H457Y and the combinations of V90I/R483C, P406L/A376T and T326I/T385N/L461Y.

The two French fusA-positive MRSA strains showed following mutations: V90I and P406L.

\section{Patient \& bacterial characteristics per resistance gene}

Patients carrying a fusB-positive MSSA strain were on average younger than patients carrying fusA- or fusC-positive S. aureus (43 years vs 54 and 53 years, respectively, $\mathrm{p}<0.01$ ). When comparing these groups on gender or having a chronic skin condition, no significant differences were found $(\mathrm{p}<0.05)$.

\section{[TABLE 1]}

Of the 25 spa typed fusB-positive isolates, 21 (84\%) were associated with spa-CC 659/171, and 17 of these 21 strains had spa t171 (associated with sequence type [ST]-123) (Table 2). For fusC strains, a large proportion (81\%; 42/52) was clustered to spa-CC 008 or CC 114 and spa t127 (associated with ST-1) was responsible for 12 of the 14 isolates of spa-CC 114. For fusA, a more diverse profile of spa-CCs was observed (Table 2).

Tetracycline resistance was significantly higher in fusC isolates as compared with the fusA/B isolates (24\% [21/88] vs $1 \%$ [1/100] respectively; $\mathrm{p}<0.01)$. The fusCpositive $\mathrm{S}$. aureus isolates that were tetracycline resistant showed no clustering by country.

\section{Multidrug resistance}

Among the fusidic acid-resistant MSSA isolates, 6/194 (3.1\%) were multidrug resistant (fusA [ $n=1]$ and fusC $[n=5]$ ). The multidrug-resistant profiles consisted mainly of tetracyclines, (tetracycline) macrolides (azithromycin/clindamycin/erythromycin) and quinolones (ciprofloxacin). For MRSA, seven out of the nine strains were multidrug-resistant, including the same antibiotic classes as for MSSA, except for one strain that showed resistance to gentamicin (fusA [n=2], fusB [n=1] and fusC $[n=4]$ ). 
Heijer, C.D.J. den, Bijnen, E.M.E. van, Paget, W.J., Stobberingh, E.E. Fusidic acid resistance in Staphylococcus aureus nasal carriage strains in nine European countries. Future Microbiology: ) 2014, 9(6), 737-745

\section{DISCUSSION}

In this study, we have evaluated nasal carriage S. aureus fusidic acid resistance, both pheno- and geno-typically. The isolates originated from GP patients from nine European countries who did not have traditional risk factors for antibiotic resistance, such as an infectious condition at inclusion, recent antibiotic use and recent hospitalization. Overall fusidic acid resistance was relatively low (2.9\%), with fusC being the predominant resistant mechanism among these fusidic acid-resistant strains. fusB and fusC were associated with specific spa-CCs, whereas fusA showed a more diverse spa profile. Moreover, fusC was associated with tetracycline resistance.

The strength of the present study is the unique, large collection of S. aureus, which gives insight in the $\mathrm{S}$. aureus fusidic acid resistance profile of nine European communities. Furthermore, standardized laboratory protocols were used for S. aureus identification and antibiotic resistance testing was performed at one central laboratory, thereby minimizing interlaboratory bias.

Our purpose was to discriminate the fusidic acid susceptible from the resistant isolates, in which determining disc diffusion zone diameters has the advantage of being feasible, although difficulties in the interpretation of the zone diameters can make the results subjective. Therefore, we verified whether isolates were correctly classified as fusidic acid resistant or susceptible based on the disc diffusion method. This was done by determining the MIC values of the isolates with zone diameters around the (disc diffusion) resistance cutoff value. The clear difference between susceptible and resistant isolates based on the MIC values shows that the resistance cutoff value we used was appropriate for this S. aureus collection.

For feasibility reasons, we chose to perform spa typing on approximately $50 \%$ of the fusidic acid-resistant S. aureus. Owing to the consistency in the spa types found per fusidic acid resistance gene, and the fact that the spa-typed isolates were randomly chosen, we think that the presented spa typing data are representative for our total collection. In this way, we believe this is a valid, cost-effective way of reporting our spa typing results

The approach to only test $\mathrm{S}$. aureus for fusA and fusE when found negative for fusB$\mathrm{D}$ was based on the paper by Castanheira et al. [2]. They stated that this approach is supported by a study by O'Neill and Chopra who found that levels of fusidic acid resistance did not increase in strains with fusA mutations after insertion of fusB [19]. In this way, strains would not benefit from a combination of resistance mechanisms, making it unlikely that such a situation occurs in clinical strains.

The highest resistance among MSSA strains was observed in the UK (7.8\%), followed by The Netherlands (5.1\%) and the lowest prevalence of resistance was observed in the participating eastern European countries, that is, Croatia (0.1\%) and Hungary (0.2\%). Unfortunately, we were not able to retrieve topical fusidic acid use data from all these countries, including an inquiry at LeoPharma (the producer of fusidic acid), which might be an explanation for these differences. Cross-resistance was addressed by Farrell et al. as another potential cause of the emergence of fusidic acid resistance, although no specific antibiotic was stated that could be involved in this cross-resistance [1]. The situation in the USA was given as an example with the presence of fusidic acid resistance without the clinical use of this agent. In this respect, the relationship found between fusC and tetracycline resistance can be of importance. Combined fusidic acid and tetracycline resistance have been reported in 
Heijer, C.D.J. den, Bijnen, E.M.E. van, Paget, W.J., Stobberingh, E.E. Fusidic acid resistance in Staphylococcus aureus nasal carriage strains in nine European countries. Future Microbiology: 2014, 9(6), 737-745

a sheep model after agricultural use of streptomycin, but the resistance mechanism for fusidic acid was not determined [20]. In this perspective, insight in the genetic elements on which fusB and fus $C$ reside is of importance. fusB has been identified on the pUB101 plasmid, which also carried genes for $\beta$-lactmase and cadmium resistance [21]. Recently, fusC has been detected on a novel staphylococcal cassette chromosome (SCC) structure, named SCCfusC, in MRSA strains. It was found upstream of the SCCmec and also included speG, probably mediating polyamine resistance [22]. Further evaluation is needed to clarify the existence of crossresistance for fusidic acid and (molecular) epidemiology of its resistance.

\section{[TABLE 2]}

In clinical S. aureus isolates, a study has shown dominance of mutational fusidic acid resistance (in MRSA) [12], while others reported a higher proportion of acquired resistance [7,23]. With most nasal carriage fusidic acid-resistant $S$. aureus strains harboring acquired resistance genes, our results imply that horizontal gene transfer is mainly responsible for $\mathrm{S}$. aureus fusidic acid resistance (present) in the community [1].

For six isolates we could not relate phenotypic resistance to the known resistance genes, implying that there is probably another genotypic variant that results in fusidic acid resistance.

Castanheira et al. investigated fusidic acid resistance in clinical Staphylococcus species from 13 European countries, including $2700 \mathrm{~S}$. aureus strains [2]. In accordance with our findings, they found that fusC was the genetic mechanism most prevalent among $\mathrm{S}$. aureus. In that study, fusA mutations were shown to be correlated with higher MIC levels than isolates in which the fusB or fusC gene was present. Amino acid alteration L461K accounted for the major part of fusA mutations in the Castanheira study, which confers high-level resistance $(\geq 512 \mathrm{mg} / \mathrm{l})$. In the present study this mutation was only found in four isolates from the UK (with MIC $>256 \mathrm{mg} / \mathrm{l}$ ). The reason for this discrepancy between nasal carriage and invasive strains needs to be explored in future studies.

In this study, the most prevalent fusA mutations (L461S and V90I) resulted in lowlevel resistance, in agreement with previous studies [1,2]. However, the combination of the latter variant with the substitution of arginine with cysteine at location 483, resulted in high-level resistance (MIC $>256 \mathrm{mg} / \mathrm{l}$ ), which has not been reported before and this also applied to the combined mutations T326I/T385N/L461Y. The other mutations causing high-level resistance, that is, P406L, H457Y and L461K, have been considered to be causally related to in vitro resistance [1,23-24]. It would be interesting to evaluate the clinical relevance of the other mutations found in this study.

For S. aureus with fusA mutations, the diverse bacterial background of resistance has been shown before [25]. Here, we showed that a large proportion (74\%) of the fusCpositive isolates belonged to spa-CC 8 or 127. The latter spa-CC was also reported by Elazhari et al. as being related to their fusC-positive clinical S. aureus strains isolated in Morocco [11]. However, they reported no clonal relationship between their fusB-positive strains, although their number was fairly low $(n=2)$. In our study, we did find a clonal relationship between fusB-positive isolates with over $80 \%$ of these strains clustering in spa-CC 159/645 for which spa type 171 was mainly responsible. This is the spa type that has also been associated with the EEFIC $[6,8]$. 
Heijer, C.D.J. den, Bijnen, E.M.E. van, Paget, W.J., Stobberingh, E.E. Fusidic acid resistance in Staphylococcus aureus nasal carriage strains in nine European countries. Future Microbiology: ) 2014, 9(6), 737-745

The EEFIC has been isolated mainly from younger patients with impetigo; in our infection-free population we did observe that, in general, fusB-positive S. aureus were isolated from younger patients than fusA- and fusC-positive S. aureus. From the countries that participated in this study, EEFIC has previously been described in clinical S. aureus strains from the UK [8], The Netherlands [6], France [26] and Sweden [27]. We also found fusB-positive isolates predominantly in these countries, implicating that the spread of this clone to other European communities is (still) limited.

For MRSA, we observed a higher prevalence of resistance to fusidic acid in comparison with MSSA isolates (9.9 vs $2.8 \%$ ), although still not alarmingly high. The majority of the fusidic acid-resistant MRSA strains were from French patients. Owing to relatively low numbers, we cannot make firm conclusions based on our data. In addition, Otter et al. stated in a review on community-associated MRSA that outbreaks of these bacteria have been described in the absence of nasal carriage [28]. Moreover, the 'European' community-acquired (CA)-MRSA clone (ST-80) is associated with resistance to fusidic acid [24], not justifying any recommendations with respect to MRSA treatment based on our nasal carriage strains.

Since it has been shown that invasive $\mathrm{S}$. aureus infections are often caused by carriage strains, our MSSA data can be applicable to GP guidelines [10]. The overall low resistance justifies the use of fusidic acid in GP patients with MSSA SSSIs in all participating countries. Nevertheless, regular surveillance needs to be performed in all participating countries to control fusidic acid resistance and to observe changes in the prevalence of resistance over time, especially with horizontal gene transfer (fusB and fusC) being the predominant cause of nasal carriage $\mathrm{S}$. aureus resistance [1].

\section{CONCLUSION}

In conclusion, $S$. aureus nasal carriage strains from nine European countries showed a low prevalence of resistance to fusidic acid, with fus $C$ being the most important genetic mechanism of this resistance. fus $B$ and fus $C$ were related to specific spa clusters, whereas fusA showed more heterogeneity. Based on antibiotic susceptibility results, fusidic acid is currently an appropriate choice for MSSA SSSIs in the GP setting of the participating countries. Owing to limited data, no recommendations can be given for MRSA infections.

\section{Future perspective}

In the past years, numerous reports have described an increase of antibiotic resistance, irrespective of country and setting. In order to control this emergence of resistance, regular surveillance is one of the options that can be performed, of which the results should be translated into clinical guidelines. With the majority of antibiotics being used outside the hospital, surveillance is essential in the outpatient setting, although at this moment most research has focused on invasive strains. With the conductance of surveillance in primary care, the use of broad-spectrum antibiotics could be limited, and hereby decreasing the enormous potential of a person's microbiota to acquire and disseminate antibiotic resistance genes. With the present study, we hope to have provided the start of an ongoing surveillance system in the participating countries and hope that similar initiatives will be undertaken in other countries. Also, more research is needed to further determine the reasons why 
Heijer, C.D.J. den, Bijnen, E.M.E. van, Paget, W.J., Stobberingh, E.E. Fusidic acid resistance in Staphylococcus aureus nasal carriage strains in nine European countries. Future Microbiology: ) 2014, 9(6), 737-745

several MRSA strains are capable of causing infection without being present at the carriage level.

EXECUTIVE SUMMARY

\section{Background}

- A person's own microbiota, including Staphylococcus aureus, is considered an important reservoir of antibiotic resistance genes.

\section{Study material}

- S. aureus strains collected from nose swabs taken from general practice patients without known risk factors for antibiotic resistance (i.e., recent antibiotic use, hospitalization) in nine European countries.

\section{Main findings}

- Overall, S. aureus resistance to fusidic acid was $2.9 \%$, ranging from $0.1 \%$ in Croatia to $7.8 \%$ in the UK.

- fus $C$ was the most commonly found genetic mechanism (46\% of all fusidic acidresistant isolates).

- spa clonal complex (spa-CC) 659/171 was associated with fusB and fusC with spa-CC 008 and 114, whereas fusA showed more heterogeneity.

- Six out of nine fusidic acid-resistant methicillin-resistant S. aureus isolates originated from France.

- Tetracycline resistance was found to be associated with fusC.

\section{Recommendations}

- Fusidic acid is currently an appropriate choice to treat methicillin-susceptible $S$. aureus (skin) infections in the GP setting of the participating countries, based on antibiotic susceptibility patterns.

- More research is needed to evaluate the appropriateness of fusidic acid in methicillin-resistant $S$. aureus skin infections general practice.

\section{Acknowledgements}

For their effort in the APRES study, the authors would like to thank the APRES Study Team: Mike Pringle, Division of Primary Care, University of Nottingham, Nottingham, UK; Herman Goossens, Laboratory of Medical Microbiology, Vaccine and Infectious Disease Institute, University of Antwerp, Antwerp, Belgium; Kathryn Hoffmann, Department of General Practice, Medical University of Vienna, Vienna, Austria; Petra Apfalter, Institute of Hygiene, Microbiology and Tropical Medicine, Elisabethinen Hospital, Linz, Austria; Stefaan Bartholomeeusen, Department of General Practice, Catholic University of Leuven, Leuven, Belgium; Greet Ieven, 
Heijer, C.D.J. den, Bijnen, E.M.E. van, Paget, W.J., Stobberingh, E.E. Fusidic acid resistance in Staphylococcus aureus nasal carriage strains in nine European countries. Future Microbiology: 2014, 9(6), 737-745

Laboratory of Medical Microbiology, Vaccine and Infectious Disease Institute, University of Antwerp, Antwerp, Belgium; Milica Katic, Department of Family Medicine, University of Zagreb, Zagreb, Croatia; Ana Budimir, Department of Clinical and Molecular Microbiology, University Hospital Centre Zagreb, Zagreb, Croatia; Gilles Hebbrecht, Department of Medical Information, French Society of General Practice, Issy-les-Moulineaux, France; László Kolozsvári, Department of Family and Occupational Medicine, University of Debrecen, Debrecen, Hungary; Jozsef Konya, Department of Medical Microbiology, University of Debrecen, Debrecen, Hungary; Joke Korevaar, NIVEL, The Netherlands Institute for Health Services Research, Utrecht, The Netherlands; Bonaventura Bolibar, Institut Universitari d'Investigació en Atenció Primària Jordi Gol, Barcelona, Spain; Elisabet Grenzner, Laboratori Clínic l'Hospitalet, Institut Català de la Salut Metropolitana Sud, l'Hospitalet, Spain; Sigvard Mölstad, Department of Clinical Sciences, Lund University, Malmö, Sweden; Andreas Matussek, Department of Laboratory Services, Ryhov County Hospital, Jönköping, Sweden; Douglas Fleming, Birmingham Research Unit, Royal College of General Practitioners, Birmingham, UK; and Andrew Lovering, Department of Medical Microbiology, North Bristol NHS Trust, Bristol, UK. The authors would also like to thank all laboratory personnel, participating GPs and their patients. Without their help this study could not have been conducted. In addition, they thank $C$ Driessen, $J$ Maes, $J$ Peeters and M Lucchesi, who performed all laboratory diagnostics at the project's central microbiological laboratory.

\section{Financial \& competing interests disclosure}

This study has been funded by the European Commission DG Research within its Seventh Framework Program (Grant Agreement 223083). The authors have no other relevant affiliations or financial involvement with any organization or entity with a financial interest in or financial conflict with the subject matter or materials discussed in the manuscript apart from those disclosed.

No writing assistance was utilized in the production of this manuscript.

\section{Ethical conduct of research}

The authors state that they have obtained appropriate institutional review board approval or have followed the principles outlined in the Declaration of Helsinki for all human or animal experimental investigations. In addition, for investigations involving human subjects, informed consent has been obtained from the participants involved.

\section{REFERENCE}

Papers of special note have been highlighted as: • of interest; •• of considerable interest

1. Farrell DJ, Castanheira M, Chopra I. Characterization of global patterns and the genetics of fusidic acid resistance. Clin. Infect. Dis. 52(Suppl. 7), S487-S492 (2011).

2. Castanheira M, Watters AA, Mendes RE, Farrell DJ, Jones RN. Occurrence and molecular characterization of fusidic acid resistance mechanisms among Staphylococcus spp. from European countries (2008). J. Antimicrob. Chemother. 65(7), 1353-1358 (2010).

- Investigated fusidic acid resistance in clinical Staphylococcus species from 13 European countries, including $2700 \mathrm{~S}$. aureus strains.

3. Craft JC, Moriarty SR, Clark K et al. A randomized, double-blind Phase 2 study comparing the efficacy and safety of an oral fusidic acid loading-dose regimen to oral 
Heijer, C.D.J. den, Bijnen, E.M.E. van, Paget, W.J., Stobberingh, E.E. Fusidic acid resistance in Staphylococcus aureus nasal carriage strains in nine European countries. Future Microbiology: ) 2014, 9(6), 737-745

linezolid for the treatment of acute bacterial skin and skin structure infections. Clin. Infect. Dis. 52(Suppl. 7), S520-S526 (2011). [CrossRef] [Medline] [CAS]

4. Guo X, Peisker K, Backbro K et al. Structure and function of fusB: an elongation factor G-binding fusidic acid resistance protein active in ribosomal translocation and recycling. Open Biol. 2(3), 120016 (2012). [CrossRef]

5. $\quad$ Lannergard J, Cao S, Norstrom T, Delgado A, Gustafson JE, Hughes D. Genetic complexity of fusidic acid-resistant small colony variants (SCV) in Staphylococcus aureus. PLoS ONE 6(11), e28366 (2011). [CrossRef] [Medline]

6. Rijnders MI, Wolffs PF, Hopstaken RM, den Heyer M, Bruggeman CA, Stobberingh EE. Spread of the epidemic European fusidic acid-resistant impetigo clone (EEFIC) in general practice patients in the south of The Netherlands. J. Antimicrob. Chemother. 67(5), 1176-1180 (2012). [CrossRef] [Medline] [CAS]

7. Castanheira M, Watters AA, Bell JM, Turnidge JD, Jones RN. Fusidic acid resistance rates and prevalence of resistance mechanisms among Staphylococcus spp. isolated in North America and Australia, 2007-2008. Antimicrob. Agents Chemother. 54(9), 3614-3617 (2010).

- Demonstrates the results of a surveillance fusidic acid resistance among invasive Staphylococcus aureus and coagulase-negative Staphylococcus species originating from North America and Australia.

8. O'Neill AJ, Larsen AR, Skov R, Henriksen AS, Chopra I. Characterization of the epidemic European fusidic acid-resistant impetigo clone of Staphylococcus aureus. J. Clin. Microbiol. 45(5), 1505-1510 (2007).

9. Andremont A, Bonten M, Kluytmans J, Carmeli Y, Cars O, Harbarth S. Fighting bacterial resistance at the root: need for adapted EMEA guidelines. Lancet Infect. Dis. 11(1), 6-8 (2011).

- Describes the relevance of taking into account the resistance present in a person's microbiota in order to control antibiotic resistance at the root.

10. Wertheim HF, Melles DC, Vos MC et al. The role of nasal carriage in Staphylococcus aureus infections. Lancet Infect. Dis. 5(12), 751-762 (2005).

11. Elazhari M, Abu-Quatouseh LF, Elhabchi D et al. Characterization of fusidic acidresistant Staphylococcus aureus isolates in the community of Casablanca (Morocco). Int. J. Med. Microbiol. 302(2), 96-100 (2012). [CrossRef] [Medline]

12. Chen HJ, Hung WC, Tseng SP, Tsai JC, Hsueh PR, Teng LJ. Fusidic acid resistance determinants in Staphylococcus aureus clinical isolates. Antimicrob. Agents Chemother. 54(12), 4985-4991 (2010).

13. van Bijnen EM, den Heijer CD, Paget WJ et al. The appropriateness of prescribing antibiotics in the community in Europe: study design. BMC Infect. Dis. 11, 293 (2011).

14. den Heijer CD, van Bijnen EM, Paget WJ et al. Prevalence and resistance of commensal Staphylococcus aureus, including meticillin-resistant $S$ aureus, in nine European countries: a cross-sectional study. Lancet Infect. Dis. 13(5), 409-415 (2013).

15. European Committee on Antimicrobial Susceptibility Testing. EUCAST Definitive Document E.DEF 3.1, June 2000: Determination of minimum inhibitory concentrations (MICs) of antibacterial agents by agar dilution. Clin. Microbiol. Infect. 6(9), 509-515 (2000). 16. BLAST. Basic Local Alignment Search Tool.http://blast.ncbi.nlm.nih.gov/Blast.cgi 17. Strommenger B, Kettlitz C, Weniger T, Harmsen D, Friedrich AW, Witte W. Assignment of Staphylococcus isolates to groups by spa typing, Smal macrorestriction analysis, and multilocus sequence typing. J. Clin. Microbiol. 44(7), 2533-2540 (2006).

18. Ridom.www.ridom.de

19. O'Neill AJ, Chopra I. Molecular basis of fusB-mediated resistance to fusidic acid in Staphylococcus aureus. Mol. Microbiol. 59(2), 664-676 (2006).

20. Scherer A, Vogt HR, Vilei EM, Frey J, Perreten V. Enhanced antibiotic multiresistance in nasal and faecal bacteria after agricultural use of streptomycin. Environ. Microbiol. 15(1), 297-304 (2013).

21. Howden BP, Grayson ML. Dumb and dumber - the potential waste of a useful antistaphylococcal agent: emerging fusidic acid resistance in Staphylococcus aureus. Clin. Infect. Dis. 42(3), 394-400 (2006).

22. Lin YT, Tsai JC, Chen HJ, Hung WC, Hsueh PR, Teng LJ. A novel staphylococcal cassette chromosomal element, SCCfusC, Carrying fusC and SpeG in fusidic acid-resistant 
Heijer, C.D.J. den, Bijnen, E.M.E. van, Paget, W.J., Stobberingh, E.E. Fusidic acid resistance in Staphylococcus aureus nasal carriage strains in nine European countries. Future Microbiology:) 2014, 9(6), 737-745

methicillin-resistant Staphylococcus aureus. Antimicrob. Agents Chemother. 58(2), 12241227 (2014).

23. McLaws FB, Larsen AR, Skov RL, Chopra I, O'Neill AJ. Distribution of fusidic acid resistance determinants in methicillin-resistant Staphylococcus aureus. Antimicrob. Agents Chemother. 55(3), 1173-1176 (2011).

24. Besier S, Ludwig A, Brade V, Wichelhaus TA. Molecular analysis of fusidic acid resistance in Staphylococcus aureus. Mol. Microbiol. 47(2), 463-469 (2003).

25. Lannergard J, Norstrom T, Hughes D. Genetic determinants of resistance to fusidic acid among clinical bacteremia isolates of Staphylococcus aureus. Antimicrob. Agents Chemother. 53(5), 2059-2065 (2009).

26. Laurent $F$, Tristan $A$, Croze $M$ et al. Presence of the epidemic European fusidic acidresistant impetigo clone (EEFIC) of Staphylococcus aureus in France. J. Antimicrob. Chemother. 63(2), 420-421; author reply 421 (2009).

27. Osterlund A, Kahlmeter G, Haeggman S, Olsson-Liljequist B; Swedish Study Group On Fusidic Acid Resistant SA. Staphylococcus aureus resistant to fusidic acid among Swedish children: a follow-up study. Scand. J. Infect. Dis. 38(5), 334-334 (2006).

28. Otter JA, French GL. Molecular epidemiology of community-associated meticillinresistant Staphylococcus aureus in Europe. Lancet Infect. Dis. 10(4), 227-239 (2010).

\section{TABLES}

Table 1. Fusidic acid resistance mechanisms among nasal carriage methicillin-susceptible Staphylococcus aureus from nine European countries.

\begin{tabular}{|c|c|c|c|c|c|c|}
\hline \multirow[t]{2}{*}{ Country } & \multirow[t]{2}{*}{$\begin{array}{l}\text { Total strains } \\
\text { tested (n) }\end{array}$} & \multirow[t]{2}{*}{$\begin{array}{l}\text { Fusidic acid } \\
\text { resistance }{ }^{\dagger}, \text { n (\%) }\end{array}$} & \multicolumn{2}{|c|}{$\begin{array}{l}\text { Acquired } \\
\text { resistance }{ }^{\ddagger}(n)\end{array}$} & \multirow[t]{2}{*}{$\begin{array}{l}\text { Acquired fusidic acid } \\
\text { resistance genes (\%) }\end{array}$} & \multirow[t]{2}{*}{ fusA mutations (no. tested) ${ }^{5}$} \\
\hline & & & fusB & fusC & & \\
\hline Austria & 541 & $5(0.9)$ & 1 & 4 & 100 & $0(0)$ \\
\hline Belgium & 570 & $19(3.3)$ & 2 & 8 & 52.6 & 2 V90I, 1 G452V, 1 H457Y, 3 L461S, 1 R464L (9) \\
\hline Croatia & 740 & $1(0.1)$ & 0 & 1 & 100 & $0(0)$ \\
\hline France & 858 & $27(3.1)$ & 3 & 20 & 85.2 & 3 V90I, 1 V90I/L461F (4) \\
\hline Hungary & 531 & $1(0.2)$ & 1 & 0 & 100 & $0(0)$ \\
\hline $\begin{array}{l}\text { The } \\
\text { Netherlands }\end{array}$ & 1064 & $54(5.1)$ & 22 & 20 & 77.8 & 4 V90I, 1 T326I/T385N/L461Y, 1 E444K, 5 L461S (12) \\
\hline Spain & 756 & $7(0.9)$ & 0 & 4 & 57.1 & 2 V90I, 1 L461S (3) \\
\hline Sweden & 955 & $18(1.9)$ & 8 & 7 & 83.3 & $\begin{array}{l}1 \text { M401T, } 1 \text { P404L, } 1 \text { isolate with } 37 \text { mutations, } \\
\text { e.g., V90I, L461F (3) }\end{array}$ \\
\hline UK & 799 & $62(7.8)$ & 11 & 24 & 56.5 & $\begin{array}{l}6 \text { V90I, } 1 \text { S95T, } 1 \text { E444K, } 1 \text { M453I, } 1 \text { H457Y, } 1 \text { L461F, } \\
4 \text { L461K, } 7 \text { L461S, } 1 \text { isolate with } 37 \text { mutations, e.g., } \\
\text { V90I (27) }\end{array}$ \\
\hline Total & 6814 & $194(2.8)$ & 48 & 88 & 70.1 & $52^{9}$ \\
\hline $\begin{array}{l}\text { 'Defined as a hav } \\
\text { ¥No fus } D \text { was det } \\
\text { 5No fusE was dete } \\
\text { 'Presents the tot: }\end{array}$ & $\begin{array}{l}\text { ing a zone diamete } \\
\text { ected among the in } \\
\text { al number of fus } A-p \text { among the in }\end{array}$ & $\begin{array}{l}\text { r }<23 \mathrm{~mm} \text { with the Kirby } \\
\text { cluded S. aureus strains. } \\
\text { cluded S. aureus strains. } \\
\text { ositive S. aureus in the pr }\end{array}$ & Bauer $r$ & $\operatorname{hod}(10-\mu$ & & \\
\hline
\end{tabular}


Heijer, C.D.J. den, Bijnen, E.M.E. van, Paget, W.J., Stobberingh, E.E. Fusidic acid resistance in Staphylococcus aureus nasal carriage strains in nine European countries. Future Microbiology: ) 2014, 9(6), 737-745

\section{Table 2. Overview of spa types and spa clonal complexes found per fus gene.}

Fusidic acid resistance Associated spa types mechanism

fusA $(\mathrm{n}=26)$

fusB $(\mathrm{n}=25)$

fusC $(\mathrm{n}=52)$ nontypeable $(n=1)$ t002, t005 ( $n=2)$, t021, t050, t091 ( $=2)$, t105, t127, t159, t189, t362, t864, t1778, t2078, t2801, t3274, t3369, t4460, t4501, t6839, t6995, t11477, nontypeable $(n=3)$ t091, t171 ( $=17), \mathrm{t377}, \mathrm{t} 408(\mathrm{n}=2), \mathrm{t659}, \mathrm{t876}, \mathrm{t7164}$, t002, t008 ( $=17)$, t015, t024 ( $=2), \mathrm{t} 114, \mathrm{t} 121, \mathrm{t} 127$ ( $n=12), \mathrm{t} 304, \mathrm{t} 386, \mathrm{t} 458, \mathrm{t} 559, \mathrm{t} 622, \mathrm{t} 656, \mathrm{t} 1508, \mathrm{t} 1888$, t2207, t3308, t4407, t6197, t6752, t11867
Associated spa-CCs (dominant)

Heterogeneous

spa-CC 659/171

spa-CC 008, spa-CC 114 